\title{
The Approach Of Cancer Related Fatigue In Rehabilitation Medicine: Part Ii - Therapeutic Interventions
}

\author{
Amalia Salca', Alexandra Checiches ${ }^{1}$, Laszlo Irsay ${ }^{1,2}$ \\ 1 Rehabilitation Clinical Hospital in Cluj-Napoca \\ 2 "Iuliu Hatieganu" UMPh in Cluj-Napoca
}

\begin{abstract}
Starting with patient' diagnose and continuing throughout the treatment and thereafter, cancer-related fatigue (CRF) is a distressing and disabling symptom, highly prevalent across the cancer continuum ${ }^{2}$.

This is a review article mainly focusing on the rehabilitation objectives and interventions in CRF, and implementation issues, according to the report of an NCCN member institution ${ }^{4}$. Implementation is the most problematic, considering the large number of patients to whom it is addressed to and the variety of pathologies within this group of patients.

The onset of CRF is difficult to establish, because of the limitations of reporting this symptom $^{4}$, but it is a valuable predictor in prognosis.

The main interventions in rehabilitation applicable to these patients are discussed in correlation to the objectives of each phase of therapeutic management in cancer: pre-operatory, before, during or after radio and/or chemotherapy
\end{abstract}

Conclusion: Rehabilitation interventions should be applied to all patients diagnosed with cancer, according to their phase of oncologic treatment and the objectives. This should be practiced as preventive measure, but as a therapeutic one to, considering the high incidence of CFR before diagnose.

\section{Introduction}

CFR is a problematic issue in cancer patient' both during as well as after treatment ${ }^{1}$. Starting with patient' diagnose and continuing throughout the treatment and thereafter, cancer-related fatigue is a distressing and disabling symptom, highly prevalent across the cancer continuum ${ }^{2}$.

According to NCCN (National Comprehensive Cancer Network) definition of fatigue, this is "a persistent subjective sense of tiredness related to cancer or cancer treatment that interferes with usual functioning " 3 . Often patients describe it as a state in which they are not able to do the kinds of activities that they used to enjoy doing ${ }^{4}$. CRF is considered one of the most significant quality of life (QOL) issues in cancer patients ${ }^{4}$.

\section{Objectives}

This is a review article mainly focusing on the rehabilitation objectives and interventions in CFR, and implementation issues, according to the report of an NCCN member institution ${ }^{4}$.

Implementation is the most problematic, considering the large number of patients to whom it is addressed to and the variety of pathologies within this group of patients.

The objective of this article is to gather the main interventions in rehabilitation applicable to these patients are discussed in correlation to the objectives of each phase of therapeutic management in cancer: preoperatory, before, during or after radio and/or chemyotherapy.

The main factors related to CRF that should be considered in cancer patients are presented here.

The topics of mechanisms, symptoms, clinical evaluation and screening in CRF are not covered by this paper, they have been detailed in the first part of this article, which was published in the issue of May 2015 of Balneo Research Journal. 


\section{Discussions}

As fatigue is mostly prevalent in other multi-systemic diseases secondary to diseaserelated - muscle disuse ${ }^{5}$, patients should be encouraged to practice constant low-intensity exercises.

In the literature it is emphasized the importance of treatment of anemia, aerobic exercise training, progressive resistance training as factors related to improving physiological functional capacity ${ }^{6}$.

In all phases of cancer treatment (preoperatory, before, during or after radio and/or chemyotherapy) anemia, cachexia, pain and sleep disorders should be assessed. Their treatment is mandatory in the attempt to lower the symptoms of CRF.
Thus, early after diagnose, the objectives

of rehabilitation programs focus on mobilization, self-care and hygiene, proper nutrition. The rehabilitation objectives are:

- Patient's physical independence: moving in bed, transfers, sitting position

- Self-care and hygiene

- Nutrition adapted to patients abilities and senses alterations

- Psychological support

- Pain control

For the pain in cancer patients, Foley classification establishes the stage of pain according to phase of cancer \& of treatment, history of chronic pain or drug abuse. Table number 1 presents the Foley classification describing each stage of pain.

\begin{tabular}{|l|l|}
\hline Foley stage & Cause of pain \\
\hline I & In acute phase or due to treatment \\
\hline II & Chronic phase, progressive evolution or due to treatment \\
\hline III & Chronic preexisting pain and pain due to cancer \\
\hline IV & History of drug abuse and pain due to cancer \\
\hline V & Terminal phase cancer associated with pain \\
\hline
\end{tabular}

Table 1: Foley Stages of pain in cancer

Aerobic conditioning and resistive exercises improve fatigue first as a direct effect and second through diminution of insomnia, nausea and emotional distress ${ }^{8}$. In practice, intensities as high as $90 \%$ maximal heart rate three times a week should be safely tolerated. But recent studies have concluded that, for example, among cancer survivors individual exercise prescription should use specific physical activity guidelines in order to maximize the benefits obtained by the use of aerobic exercise training ${ }^{9}$ Therefore the physical guidelines used for healthy subjects need to be adapted.

There are evidences which show that a complex management of CRF- involving exercise, psycho-educational counselling, and cognitive-behavioral therapy for insomnia - is effective in this category of patients ${ }^{2}$.
Because CRF is often not revealed in patients and doctors' reports, most patients do not undergo the CRF management. A study conducted in an NCCN member Institution showed that the implementation of Fatigue Guidelines is difficult due to different type of barriers. These can be classified as patient-, provider- and system- related barriers. The most frequent patient-related barrier in reporting CRF symptoms and in receiving indications for the treatment of fatigue, was patient's belief that the doctor would introduce this subject if it was important ${ }^{4}$.

Special categories of patients are the ones with cardiopulmonary dysfunction, bone metastasis, lymph-oedema or radiation contractures.

Patients with cancer should be assessed for cardiopulmonary dysfunction if their 
pathology is related or affecting the respiratory system or thoracic wall and cavity. This can be due to resection or radiation of the lung tissue.

In patients with bone metastasis, clinicians should assess whether a weightbearing bone is involved. For these patients the following are recommended: assistive devices, use of orthoses, environmental modifications and therapeutic exercises. Within the physical program, the prescription must include postural and balance training and truncal strengthening ${ }^{8}$.

The Lymphoedema management is done through complex decongestive therapy $(\mathrm{CDT})^{8}$ which includes: manual lymphatic drainage (MLD), short stretch compressive bandaging, skin care, therapeutic exercise, elastic compression garments. CDT has two phases: one (I) for volume reduction and second (II) for maintenance. All the way throughout the program, compression is the basis of therapy.

Contractures due to local radiation have successful outcomes with passive and active stretching. The flexibility exercises should be carefully prescribe and as soon as possible.

\section{Conclusion}

Rehabilitation programs should be adapted to patient and must address the medical issues accurately. The physical modalities used in these patients cover a wide range: assistive devices, use of orthoses, environmental modifications, nutritional interventions and therapeutic exercises.

Early mobilization is important and along with flexibility exercise can be carefully applied from the early phases. Further, aerobic and resistive exercises should be individually prescribed according to patient abilities.

Physical modalities can enhance the outcome of cancer patients with CRF, because it can address in a complex way many issues within it.

\section{References:}

1.Roscoe JA, Kaufman ME, Matteson-Rusby SE, Palesh OG, Ryan JL, Kohli S, Perlis ML, Morrow GR. Cancer-related fatigue and sleep disorders. Oncologist. 2007; 12 Suppl 1:35-42. 2. Mitchell SA. Cancer-related fatigue: state of the science. PM R. 2010 May; 2(5):364-83.

3. Wang XS, Zhao F, Fisch MJ, O'Mara AM, Cella D, Mendoza TR, Cleeland CS. Prevalence and characteristics of moderate to severe fatigue: a multicenter study in cancer patients and survivors. Cancer. 2014 Feb 1; 120(3):425-32. Fulltext available at: http://onlinelibrary.wiley.com/doi/10.1002/ cncr.28434/pdf

4. Borneman T, Piper BF, Sun VC, Koczywas M, Uman G, Ferrell B. Implementing the Fatigue Guidelines at one NCCN member institution: process and outcomes. J Natl Compr Canc Netw. 2007 Nov; 5(10):1092-101. Fulltext available at: http://www.ncbi.nlm.nih.gov/pmc/articles/ PMC2515169/

5.Maughan D, Toth M. Discerning primary and secondary factors responsible for clinical fatigue in multisystem diseases. Biology (Basel). 2014 Sep 22; 3(3):606-22. Fulltext available at: http://www.mdpi.com/20797737/3/3/606

6.Evans WJ. Physical function in men and women with cancer. Effects of anemia and conditioning. Oncology (Williston Park). 2002 Sep; 16 (9 Suppl 10): 109-15. Fulltext available at: http://www.cancernetwork.com/oncologyjournal/physical-function-men-and-women-

cancer

7.Tache G.O., "Guide for Physical Medicine and Medical Rehabilitation”, 2001.

8.Braddom RL. et all, "Physical Medicine and Rehabilitation" $4^{\text {th }}$ Edition.

9.Gil-Rey E, Quevedo-Jerez K, MaldonadoMartin S, Herrero-Román F. Exercise Intensity Guidelines for Cancer Survivors: a Comparison with Reference Values. Int J Sports Med. 2014 Nov 27. 